Published in "Natural Product Research 31 (24): 2875-2884, 2017"

which should be cited to refer to this work.

\title{
A new procyanidin B from Campylospermum zenkeri (Ochnaceae) and antiplasmodial activity of two derivatives of $( \pm)$-serotobenine
}

\begin{abstract}
Norbert Mbabi Nyemeck II a,c, Dominique Serge Ngono Bikobo a , Auguste Abouem A Zintchem $^{\mathrm{a}, \mathrm{b}}$, Eva-Maria Schäfer ${ }^{\mathrm{d}}$, Christian Bochet ${ }^{\mathrm{e}}$, Dieudonné Emmanuel Pegnyemb ${ }^{a}$ and Ulrich Koert ${ }^{c}$

aDepartment of Organic Chemistry, Faculty of Science, University of Yaoundé I, Yaoundé, Cameroon; bepartment of Chemistry, Higher Training College, University of Yaoundé I, Yaoundé, Cameroon; 'Faculty of

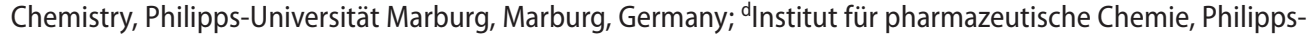

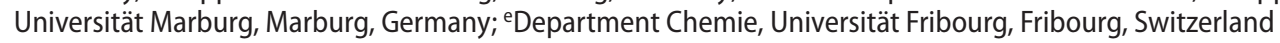

\begin{abstract}
Phytochemical investigation of the stem bark of Campylospermum zenkeri led to the isolation of five known compounds: $(Z, Z)-9,12-$ octadecadienoic acid (1), serotobenine (2), agathisflavone (3), lophirone A (4) and lophirone $F(5)$, together with a new derivative of procyanidin B, a catechin dimer named zenkerinol (6). Serotobenine (2) is structurally related to decursivine which shows moderate activity against D6 and W2 strains of Plasmodium falciparum. For a better understanding of structure-activity relationships, three new semisynthetic derivatives of serotobenine (2) have been prepared. These are: serotobenine monopropionate (2a), serotobenine monopivalate (2b) and serotobenine cyclohexyl ether (2c) respectively. Two of them (2a) and (2b), were evaluated for their antiplasmodial activity against $P$. falciparum 3D7 strain in a parasite lactate-dehydrogenase (pLDH) assay. Compound $\mathbf{2} \mathbf{b}$ was more active than compound $\mathbf{2} \mathbf{a}$ based on their $I C_{50}$ values (36.6 and $123 \mu \mathrm{M}$, respectively).
\end{abstract}

\section{KEYWORDS}

Campylospermum zenkeri; Ochnaceae; zenkerinol; flavonoids; serotobenine; hemisynthesis; antiplasmodial activity

CONTACT Dominique Serge Ngono Bikobo ngono_serge@yahoo.fr

(6) Supplemental data for this article can be accessed at http://dx.doi.org/10.1080/14786419.2017.1305378. 

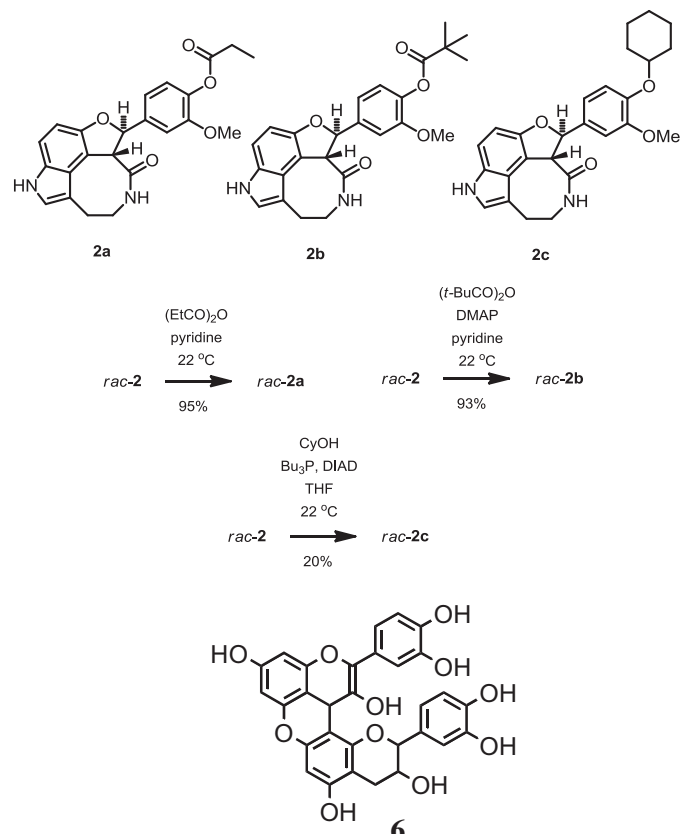

\section{Introduction}

Malaria is the most prevalent disease in the world, killing about 1-2 million people each year. It is a threat to approximately 3.4 billion people in about 209 countries and territories (Diarra et al. 2015). In 2012, there were an estimated 207 million cases of malaria with over 627.000 deaths (World Health Organization 2014). Sub-Sahara Africa carries the biggest burden of this disease, where about $90 \%$ of cases and death occur particularly among children and pregnant women (Chinsembu 2015) due to poverty and debilitation. Increasingly, malarial parasites, particularly P. falciparum, are developing resistance to the most frequently used antimalarial drugs (White 2004). The challenge in malaria chemotherapy is to find novel molecular targets in light of the drug resistance crisis (Frederich et al. 2008). In this context, the search for new compounds from Cameroonian medicinal plants could provide new leads to antimalarial drugs. Plants of the genus Campylospermum (Ochnaceae) are widely distributed throughout the tropical zone of Africa Continent. Several species belonging to this genus are reported in folk medicine in the treatment of various diseases such as gastric pains, gonorrhoea, icterus, whitlow, malaria and aphrodisiac matter (Bouquet 1969; Ngono et al. 2011, 2015). Previous reports indicated that the genus and many others belonging to Ochnaceae family are a rich source of compounds with wide variety of structures (Abouem à Zintchem et al. 2008; Abouem et al. 2014; Bayiha Ba Njock et al. 2013; Ghogomu et al. 1990; Ndongo et al. 2010, 2015; Ngo Mbing et al. 2014; Ngono Bikobo et al. 2011, 2014, 2015). Hence, this study was carried out to isolate and elucidate the structure of constituents from stem bark extract of $C$. zenkeri, then to perform few chemical transformations on an isolated one serotobenine (which is the marker of the 
genus) in order to evaluate the potent bioactive derivative for antiplasmodial activity. Serotobenine was chosen for derivatisation because of its structural similarity to decursivine, which has shown antimalarial properties in previous studies (Zhang et al. 2002; Qin et al. 2011).

\section{Results and discussion}

The methanolic extract of the stem bark of $C$. zenkeri was fractionated and purified by a silica gel column chromatography, affording five known compounds: (Z,Z)-9,12-octadecadienoic acid (1) (Park et al. 2011), racemic serotobenine (2) (Ngono Bikobo et al. 2015; Sato et al. 1985), agathisflavone (3) (Mashima et al. 1970; Bayiha Ba Njock et al. 2011), lophirone A (4) (Ghogomu et al. 1987) and lophirone F (5) (Ghogomu et al. 1990) together with one newly described compound, zenkerinol (6, Figure 1). In addition to these compounds, three new serotobenine derivatives were prepared by using racemic serotobenine (2) as starting material (Scheme 1). They are: serotobenine monopropionate (2a), serotobenine monopivalate (2b) and serotobenine cyclohexyl ether (2c). While in decursivine, two phenolic hydroxyl groups are part of a methylenedioxo-acetal substructure, it seemed reasonable to protect the free hydroxyl group of serotobenine (2) in order to explore the importance of the acetal for the antiplasmodial activity. Therefore, the free phenolic group was covered as ester structures (Figure S1 and S2).

Compound $\mathbf{6}$ was obtained as an orange powder, soluble in methanol. The molecular formula of this compound was inferred to be $\mathrm{C}_{30} \mathrm{H}_{22} \mathrm{O}_{11}$ based on its molecular ion at $\mathrm{m} / \mathrm{z}$ $557.1089[\mathrm{M}-\mathrm{H}]^{-}$in negative ion mode HR-ESI-MS (calcd. 557.1131). The ${ }^{13} \mathrm{C}$ NMR spectral data of $\mathbf{6}$ (Table S2) revealed that this compound is a procyanidin derivative with two condensed flavonoids. That presence of two flavonoid units was indicated by ${ }^{13} \mathrm{C}$ NMR resonances at $\delta_{C} 140.0(C-2), 133.1(C-3), 48.8$ (C-4) (ring C) which corresponds to deprotonated catechin and at $\delta_{C} 78.3\left(C-2^{\prime \prime}\right), 65.1\left(C-3^{\prime \prime}\right), 28.5\left(C-4^{\prime \prime}\right)$ (ring F), arising from flavanyl and flavenyl heterocyclic rings, respectively. These signals provide a distinct key entry point into the two-dimensional spectra (Ragab et al. 2013). Along with the absence of H-8" of D-ring in the flavan unit (sub-structure II, Figure S3), the above-mentioned signals indicated that this compound is a condensed flavonoid of procyanidin B series (Cádiz-Gurrea et al. 2014; Klika et al. 2015). The attachment of proton signals at $\delta_{\mathrm{H}} 3.18(\mathrm{H}-4)$, in sub-structure $I$ to the carbon atom at $\delta_{\mathrm{C}}$ 48.8 (C-4) ppm was noted in the HSQC spectrum (Figure S13). The same observations are found for signals at $\delta_{\mathrm{H}} 4.65\left(\mathrm{H}-2^{\prime \prime}\right), 3.91\left(\mathrm{H}-3^{\prime \prime}\right), 2.66$ and $2.46\left(\mathrm{H}-4^{\prime \prime}\right)$ with carbons at $\delta_{\mathrm{C}} 78.3$ $\left(\mathrm{C}-2^{\prime \prime}\right), 65.1\left(\mathrm{C}-3^{\prime \prime}\right)$ and $28.5\left(\mathrm{C}-4^{\prime \prime}\right)$, respectively in sub-structure II, which were consistent with the terminal unit of a catechin moiety (Figure S3) (Mello de et al. 1999; Wang et al. 2015). In the ${ }^{1} \mathrm{H}$ NMR spectrum, the set of meta-coupled protons at $\delta_{\mathrm{H}} 5.88(1 \mathrm{H}, \mathrm{d}, J=2.3 \mathrm{~Hz})$ and 5.71 $(1 \mathrm{H}, \mathrm{d}, J=2.3 \mathrm{~Hz})$ was, respectively, assigned to $\mathrm{H}-6$ and $\mathrm{H}-8$ protons of the A-ring of the sub-structure I, while a residual aromatic proton singlet appeared at $\delta_{H} 6.18\left(\mathrm{~s}, \mathrm{H}-6^{\prime}\right)$ from $D$ ring. The HMBC spectrum (Figure S14) of compound 6 revealed correlation of the signal at $\delta_{\mathrm{H}} 5.88(\mathrm{H}-6)$ and carbons at $\delta_{\mathrm{C}} 156.5$ (C-7), 99.9 (C-4a) 94.4 (C-8) and 156.0 (C-5) (Figure S3). The $\mathrm{C}-8^{\prime \prime}$ involvement in the interflavan lineage was construed from the HMBC correlations, which enabled us to assign the $\mathrm{C}-8^{\prime \prime}$ carbon atom. From the HMBC spectrum, correlations were observed between $\mathrm{H}-4\left(\delta\right.$ 3.18) with $C-8^{\prime \prime}(\delta 102.4), C-7^{\prime \prime}(\delta 156.0)$ and $\mathrm{C}-3$ ( $\delta$ 133.1) confirming the $\mathrm{C}-4 \rightarrow \mathrm{C}-8$ " bond. Moreover, comparison of the high chemical shift values of C-4a and C-8" at $\delta_{C} 99.9$ and 102.4 with literature data (Malan et al. 1996; Messanga et al. 
<smiles>CCCCC/C=C\C/C=C\CCCCCCCC(=O)O</smiles>

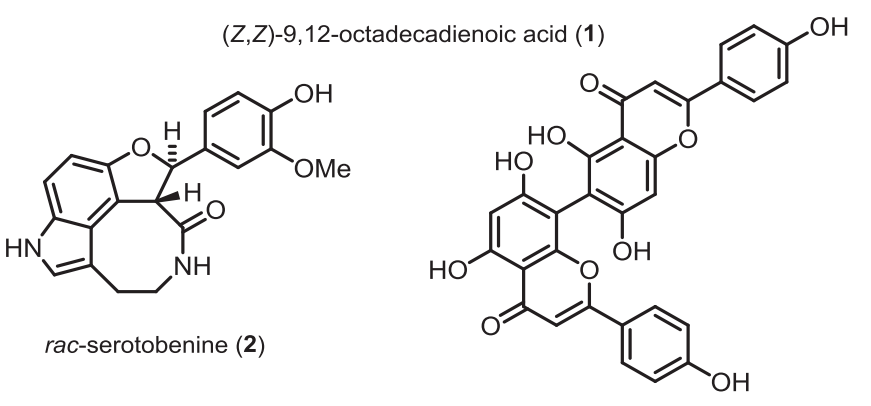

agathisflavone (3)<smiles>O=C(c1ccc(O)cc1O)[C@@H]1[C@H](c2ccc(O)cc2)O[C@H](c2ccc(O)cc2)[C@@H]1C(=O)c1ccc(O)cc1O</smiles>

lophirone F (5)

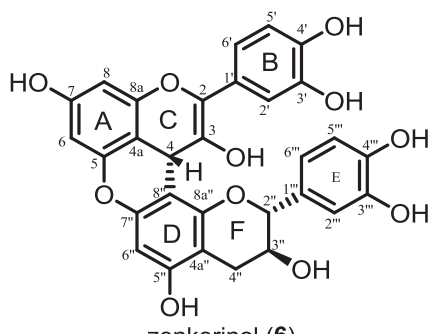

zenkerinol (6)<smiles>O=C(c1ccc(O)cc1O)C(c1ccc(O)cc1)(c1ccc(O)cc1)c1coc2cc(O)ccc12</smiles>

lophirone A (4)

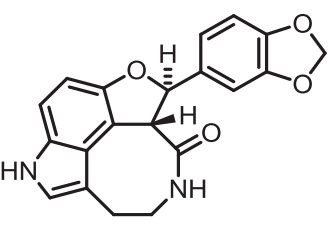

(-)-decursivine

Figure 1. Structures of natural products 1-6 isolated from the stem bark of Campylospermum zenkeri and antiplasmodial template decursivine.

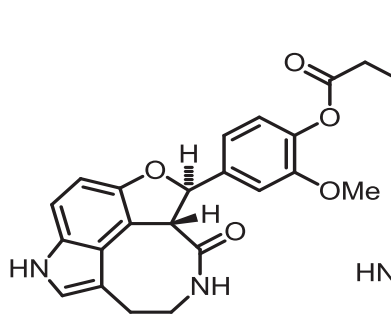

$2 \mathbf{a}$

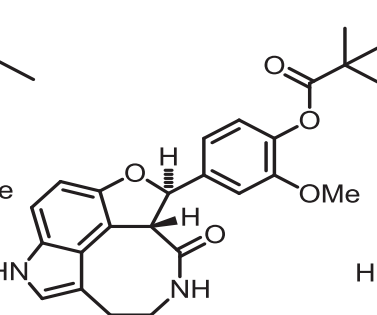

2b<smiles></smiles>

2c

$$
\begin{aligned}
& (\mathrm{EtCO})_{2} \mathrm{O} \quad(t-\mathrm{BuCO})_{2} \mathrm{O} \\
& \text { pyridine pyridine }
\end{aligned}
$$

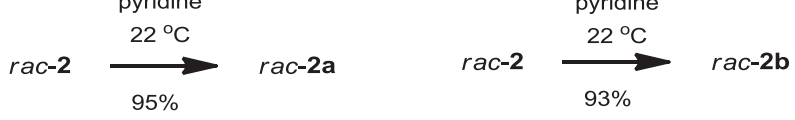

$$
\begin{aligned}
& \mathrm{CyOH} \\
& \mathrm{Bu}_{3} \mathrm{P}, \mathrm{DIAD} \\
& \text { THF } \\
& \text { rac-2 } \underset{20 \%}{\stackrel{22}{\circ} \mathrm{C}} \mathrm{rac}-2 \mathrm{c}
\end{aligned}
$$

Scheme 1 . Synthesis of three serotobenine derivatives $2 a, 2 b$ and $2 c$. 
1998; Mello de et al. 1999; Taniguchi et al. 2007; Ragab et al. 2013; Wang et al. 2015) and referring to mass spectrometry (HR-ESI-MS and LC-MS) of $\mathbf{6}$ (Figure S15; S16) indicated the presence of a C-O-C linkage, which are characterized by the existence of a doubly interflavanoid linkage. This doubly linked structure was also supported through HMBC spectrum. This was confirmed by correlations between $\mathrm{H}-6^{\prime \prime}\left(\delta_{H} 6.18\right)$ and the hyperconjugated carbon at $\delta_{C} 102.4\left(C-8^{\prime \prime}\right)$ and $\delta_{C} 156.0\left(C-7^{\prime \prime}\right)$ in one side for the second unit, and $\mathrm{H}-6\left(\delta_{H} 5.88\right)$ and the carbon $C-5\left(\delta_{C} 156.5\right)$ in another side. Moreover, this linkage was strengthened by the analysis of NOESY spectrum (Figure S17) through cross-peaks observed between $\mathrm{H}-6^{\prime \prime}\left(\delta_{\mathrm{H}} 6.18\right)$ and $\mathrm{H}-6\left(\delta_{\mathrm{H}} 5.88\right)$. Additional data from the HMBC spectrum, enabled us to locate $\mathrm{C}-2$ at $\delta_{C} 140.0$, $C-1^{\prime}$ at $\delta_{C} 120.2$ [through correlations between $\mathrm{H}-6^{\prime}\left(\delta_{H} 6.89\right)$ and olefinic carbons at $\delta_{C} 140.0$ $(C-2)$ and $C-1^{\prime}(120.2)$, respectively] and $C-3\left(\delta_{C} 133.1\right)$ involved in a relationship with $\mathrm{H}-4\left(\delta_{\mathrm{H}}\right.$ 3.18); these data were consistent with a flav-2-en-3-ol type flavonoid (Fukami et al. 2013). Consequently, all those various details emphasize the presence of another flavonoid linkage for this molecule. It was noted that the flav-2-en-3-ol moiety was found as intermediate in the synthesis of anthocyanin (Fukami et al. 2013). In addition, IR spectrum (Figure S10) at $1595 \mathrm{~cm}^{-1}$ also confirmed the presence of a conjugated olefinic double bond in this molecule (Marković et al. 2013). Signals for ABX coupling system $\left[\delta_{\mathrm{H}} 6.89\left(1 \mathrm{H}, \mathrm{d}, J=1.7 \mathrm{~Hz}, \mathrm{H}-2^{\prime}\right) ; \delta_{\mathrm{H}}\right.$ $6.66\left(1 \mathrm{H}, \mathrm{dd}, J=7.2 ; 1.7 \mathrm{~Hz}, \mathrm{H}-6^{\prime}\right)$ and $\left.\delta_{\mathrm{H}} 6.65\left(1 \mathrm{H}, \mathrm{d}, J=7.2 \mathrm{~Hz}, \mathrm{H}-5^{\prime}\right)\right]$ of sub-structures I and II of the terminal unit (due to rings $B$ and E) were observed (Table S2). These findings indicated that compound $\mathbf{6}$ was a B-type procyanidin dimer derivative consisting of a flav-2-en-3-ol and flavan-3-ol units. Further evidence to support the proposed structure could be seen in HMBC spectrum (Figure S14), some observations were made between hydroxyl protons $\mathrm{OH}-3^{\prime}$ at $\delta_{H} 8.80$ with $C-3^{\prime}\left(\delta_{C} 144.8\right)$ and $\mathrm{OH}-4^{\prime}\left(\delta_{H} 8.74\right)$ with $C-4^{\prime}\left(\delta_{C} 144.7\right)$, indicating an ortho position of these groups in the molecule (Figure $S 3$ ). These finding confirmed that cyanidin derivative and catechin are the basic skeleton of this molecule with loosing of a $\mathrm{H}_{2} \mathrm{O}$ molecule to form a supplementary pyranic cycle as described above. The coupling constants of protons of the cyclohexyl units indicated, however, clearly the trans-relationships between $\mathrm{H}-2^{\prime \prime}\left(\delta_{\mathrm{H}}\right.$ $4.65 ; J=9.5 \mathrm{~Hz}$ ) and $\mathrm{H}-3^{\prime \prime}\left(\delta_{\mathrm{H}} 3.91 ; J=9.5 ; 4.2 \mathrm{~Hz}\right)$ (Table S2). All these results are confirmed by any correlation in the NOESY spectrum (Figure S17) between neither $\mathrm{H}-2^{\prime \prime}$ and $\mathrm{H}-3$ " nor $\mathrm{H}-4$ and $\mathrm{H}-3^{\prime \prime}$, respectively. These data corroborated the relative stereochemistry found for other analogues described in the literature (Messanga et al. 1998; Mello de et al. 1999; CádizGurrea et al. 2014; Klika et al. 2015). The additional data emphasized on the double linkage between the two flavonoid units. Based on these spectral evidences and comparison with reported data, the structure of 6 was therefore determined as $\left(4 S^{*}, 2^{\prime \prime} R^{*}, 3^{\prime \prime} S^{*}\right)$-cyanidin$(4 \alpha \rightarrow 8)-\left(5-0-7^{\prime \prime}\right)$-catechin, named zenkerinol. To our knowledge, this is the first paper reporting the isolation of a procyanidin dimer in the Campylospermum genus.

Serotobenine monopropionate (2a), serotobenine monopivalate (2b) and serotobenine cyclohexyl ether (2c) were prepared according to Scheme 1, and their structures were characterized based on the spectroscopic data mentioned in supplementary material.

Serotobenine derivatives $\mathbf{2} \mathbf{a}$ and $\mathbf{2} \mathbf{b}$ were tested for their antimalarial activity against $P$. falciparum strain 3D7 in a parasite lactate dehydrogenase ( $p L D H)$-Assay. Both derivatives were less potent than decursivine. For decursivine, an antimalarial activity of 3.9 $\mu \mathrm{M}$ (D6-P. falciparum clone sensitive to chloroquine) has been reported (Zhang et al. 2002). Compound $\mathbf{2 b}$ has an $\mathrm{IC}_{50}$ value of $36.6 \mu \mathrm{M}$ and is about 10 times less active than decursivine, but is approximately 3.37 -fold better than compound $2 \mathbf{2 a}(123 \mu \mathrm{M})$. The bulky tert-butyl moiety leads to a slight increase of the antimalarial activity. Moreover, according to the Basco et al. 
(1994) criteria, the activity against $P$. falciparum can be considered moderate for compound $\mathbf{2 b}$ and low for compound $\mathbf{2 a}$ (Table S3).

\section{Experimental section}

\subsection{General procedures}

Melting points were uncorrected and were measured on a Mettler Toledo instrument. IR spectra were recorded on an Alpha FT-IR Spectrometer from Bruker, while 1D and 2D NMR spectra were obtained on a Bruker DRX $500\left(500 \mathrm{MHz}\right.$ for ${ }^{1} \mathrm{H}$ and $125 \mathrm{MHz}$ for ${ }^{13} \mathrm{C}$ spectra) spectrometer (Bruker, Rheinstetten, Germany) with chemical shifts reported in $\delta(\mathrm{ppm})$ using TMS $\left(\delta_{H}\right)$ as an internal standard. The HR-ESI-MS were obtained on LTQ-FT instrument (Thermo Scientific). LC-MS were measured with Shimadzu LC-MS system. Optical rotations were measured on a Perkin-Elmer 341 polarimeter. Silica gel 60 (230-400 mesh E. Merck, Darmstadt, Germany) was employed for column chromatography, the solvent mixing systems for elution were mainly $\mathrm{CH}_{2} \mathrm{Cl}_{2} / \mathrm{MeOH}$ for the phytochemical study and $\mathrm{CHCl}_{3} / \mathrm{MeOH}$ for the purity of compounds from hemisynthesis reactions with increasing polarity each. All reactions sensitive to oxygen or moisture were conducted under argon atmosphere.

\subsection{Plant material}

Stem barks of $C$. zenkeri were collected at Pala Mount near Kribi in the South Region of Cameroon in April 2015 and identified by a botanist. A voucher specimen ( $N^{\circ} 24093$ SFRCAM) was deposited at the National Herbarium in Yaoundé, Cameroon.

\subsection{Extraction and isolation}

Dried and powered stem bark of $C$. zenkeri $(315 \mathrm{~g})$ were extracted for $48 \mathrm{~h}$ with $\mathrm{MeOH}(3 \times 1 \mathrm{~L})$ at room temperature. After filtration and evaporation of solvent, the crude $\mathrm{MeOH}$ extract $(20 \mathrm{~g})$ was subjected to $\mathrm{CC}\left(\mathrm{SiO}_{2}\right.$, eluting with a gradient solvent system $\left.\left(\mathrm{CH}_{2} \mathrm{Cl}_{2} / \mathrm{MeOH}\right)\right)$ giving five main fractions: I (3 g), II (4 g), III (4 g), IV (4 g), V (5 g). Fraction III (4 g) was submitted to $\mathrm{CC}\left(\mathrm{SiO}_{2}\right)$ using solvent system pentane/ethyl acetate (40/1) to give compound $\mathbf{1}(120 \mathrm{mg})$. Fraction IV (4 g) was submitted to CC ( $\left.\mathrm{SiO}_{2}\right)$ using solvent system $\mathrm{CH}_{2} \mathrm{Cl}_{2} / \mathrm{MeOH}(60 / 1$ to $5 / 1)$ to give three sub-fractions (IVa, IVb and IVc). Sub-fraction IVb $(0,8 \mathrm{~g})$ was chromatographed $\left(\mathrm{SiO}_{2}\right)$ using $\mathrm{CH}_{2} \mathrm{Cl}_{2} / \mathrm{MeOH}(40 / 1)$ to afford compound 2 (35 mg; $[\alpha]_{D}^{25}=0$ (c $\left.0.15, \mathrm{MeOH}\right)$. Using the same process, fraction $\mathrm{V}(5 \mathrm{~g})$ gave 4 sub-fractions ( $\mathrm{Va}, \mathrm{Vb}, \mathrm{Vc}$ and $\mathrm{Vd})$. Sub-fraction $\mathrm{Va}(1,3 \mathrm{~g})$ was further chromatographed on a silica gel column using $\mathrm{CH}_{2} \mathrm{Cl}_{2} / \mathrm{MeOH}(10 / 1)$ to afford compound $\mathbf{3}(13 \mathrm{mg})$. Sub-fraction $\mathrm{Vb}(1.8 \mathrm{~g})$ was purified by repeated CC on silica gel with the solvent system $\mathrm{CH}_{2} \mathrm{Cl}_{2} / \mathrm{MeOH}(10 / 1-5 / 1)$ to provide compound 4 (7 mg) and compound $5(6 \mathrm{mg})$. Sub-fraction $\mathrm{Vc}(0.85 \mathrm{~g})$ was subjected to $\mathrm{CC}\left(\mathrm{SiO}_{2}\right)$ with the system $\mathrm{CH}_{2} \mathrm{Cl}_{2} / \mathrm{MeOH}(5 / 1-3 / 1)$ and compound $6\left(5 \mathrm{mg} ;[\alpha]_{D}^{25}=-79^{\circ}\right.$ (c $\left.0.3, \mathrm{MeOH}\right)$ was obtained.

\subsection{Structural characterisation of compounds}

\subsubsection{Serotobenine monopropionate (2a)}

White solid, (5.5 mg, 95\%) (Scheme 1). $\mathrm{Rf}=0.44\left(\mathrm{CHCl}_{3} / \mathrm{MeOH}: 15: 1\right)$; m.p. $250-252^{\circ} \mathrm{C}$; UV/ Vis (MeOH): $\lambda_{\max } 336$ (log e): 206 (4.50), 204 (3.44), 201 (4.50) nm; IR cm ${ }^{-1}$ 3333, 2940, 1755, 
$1659,1604,1577,1511 ;{ }^{1} \mathrm{H}$ NMR (acetone- $\left.d_{6} ; 500 \mathrm{MHz}\right), \delta_{\mathrm{H}}: 10.17(1 \mathrm{H}, \mathrm{br}, \mathrm{H}-1), 8.01(1 \mathrm{H}$, br, $\mathrm{H}-10), 7.27\left(1 \mathrm{H}, \mathrm{dd}, J=9.3 \mathrm{~Hz}, \mathrm{H}-6^{\prime}\right), 7.25\left(1 \mathrm{H}, \mathrm{d}, \mathrm{H}-2^{\prime}\right), 7.18(1 \mathrm{H}, \mathrm{s}, \mathrm{H}-2), 7.09(1 \mathrm{H}, \mathrm{d}, J=7.0 \mathrm{~Hz}$, $\mathrm{H}-7), 7.07(1 \mathrm{H}, \mathrm{d}, J=7.0 \mathrm{~Hz}, \mathrm{H}-6), 6.72\left(1 \mathrm{H}, \mathrm{d}, J=9.3 \mathrm{~Hz}, \mathrm{H}-5^{\prime}\right), 6.40\left(1 \mathrm{H}, \mathrm{d}, J=10.5 \mathrm{~Hz}, \mathrm{H}-7^{\prime}\right)$, $4.78\left(1 \mathrm{H}, \mathrm{d}, J=10.5 \mathrm{~Hz}, \mathrm{H}-8^{\prime}\right), 4.17(1 \mathrm{H}, \mathrm{m}, \mathrm{H}-9 \mathrm{~b}), 3.82\left(3 \mathrm{H}, \mathrm{s}, \mathrm{CH}_{3} \mathrm{O}-\right), 3.58(1 \mathrm{H}, \mathrm{m}, \mathrm{H}-9 \mathrm{a})$, 3.10-3.05 (1H, m, H-8a), 3.18-3.15 (2H, m, H-8b), $2.58\left(2 \mathrm{H}, \mathrm{q}, \mathrm{H}-2^{\prime \prime}\right), 1.20\left(3 \mathrm{H}, \mathrm{t}, \mathrm{H}-3^{\prime \prime}\right) ;{ }^{13} \mathrm{C} \mathrm{NMR}$ (acetone- $\left.d_{\sigma^{\prime}} ; 25 \mathrm{MHz}\right), \delta_{\mathrm{C}}$ : $172.6\left(\mathrm{C}-1^{\prime \prime}\right), 171.4\left(\mathrm{C}-9^{\prime}\right), 153.6\left(\mathrm{C}-3^{\prime}\right), 152.4(\mathrm{C}-5), 144.8(\mathrm{C}-7 \mathrm{a})$, 141.9 (C-1'), $134.1\left(\mathrm{C}-4^{\prime}\right), 125.9$ (C-3a), 125.6 (C-6'), 124.9 (C-2), 123.6 (C-6), 119.1 (C-7), 115.0 (C-4), $112.3(\mathrm{C}-3), 111.3\left(\mathrm{C}-2^{\prime}\right), 105.4\left(\mathrm{C}-5^{\prime}\right), 85.0\left(\mathrm{C}-7^{\prime}\right), 56.3\left(\mathrm{CH}_{3} \mathrm{O}-\right), 55.4\left(\mathrm{C}-8^{\prime}\right), 41.5(\mathrm{C}-9 \mathrm{a} ; 9 \mathrm{~b})$, 30.7 (C-8a; 8b), 27.6 (C-2"), 9.4 (C-3"); HR-ESI-MS m/z: $405.4307[\mathrm{M}-\mathrm{H}]^{-}$(calcd for $\mathrm{C}_{23} \mathrm{H}_{22} \mathrm{~N}_{2} \mathrm{O}_{5}$ 405.4303).

\subsubsection{Serotobenine monopivalate ( $2 \mathrm{~b})$}

White solid, (5.8 mg, 93\%) (Scheme 1). $\mathrm{Rf}=0.38\left(\mathrm{CHCl}_{3} / \mathrm{MeOH}: 30: 1\right) ; \mathrm{m} . \mathrm{p} .269-272{ }^{\circ} \mathrm{C}$; UV/ Vis (MeOH): $\lambda_{\max } 336$ (log e): 206 (4.50), 204 (3.44), 201 (4.50) nm; IR cm ${ }^{-1}: 3427,3344,3212$, $2963,2904,1738,1658,1605,1579,1509 ;{ }^{1} \mathrm{H}$ NMR (acetone- $\left.d_{6} ; 00 \mathrm{MHz}\right) ; \delta_{\mathrm{H}}: 10.21(1 \mathrm{H}$, br, $\mathrm{H}-1), 8.03(1 \mathrm{H}, \mathrm{br}, \mathrm{H}-10), 7.29\left(1 \mathrm{H}, \mathrm{dd}, J=9.3 \mathrm{~Hz}, \mathrm{H}-6^{\prime}\right), 7.25\left(1 \mathrm{H}, \mathrm{d}, \mathrm{H}-2^{\prime}\right), 7.19(1 \mathrm{H}, \mathrm{s}, \mathrm{H}-2), 7.10$ $(1 \mathrm{H}, \mathrm{d}, J=7.0 \mathrm{~Hz}, \mathrm{H}-7), 7.08(1 \mathrm{H}, \mathrm{d}, J=7.0 \mathrm{~Hz}, \mathrm{H}-6) ; 6.74\left(1 \mathrm{H}, \mathrm{d}, J=9.3 \mathrm{~Hz}, \mathrm{H}-5^{\prime}\right), 6.41(1 \mathrm{H}, \mathrm{d}$, $\left.J=10.5 \mathrm{~Hz}, \mathrm{H}-7^{\prime}\right), 4.78\left(1 \mathrm{H}, \mathrm{d}, J=10.5 \mathrm{~Hz}, \mathrm{H}-8^{\prime}\right), 4.17-4.12(1 \mathrm{H}, \mathrm{m}, \mathrm{H}-9 \mathrm{~b}), 3.83\left(3 \mathrm{H}, \mathrm{s}, \mathrm{CH}_{3} \mathrm{O}-\right)$, 3.60-3.57 (1H, m, H-9a), 3.16-3.15 (1H, m, H-8a), 3.12-3.09 (2H, m, H-8b), 1.35 (9H, s, H-3"); ${ }^{13} \mathrm{C}$ NMR (acetone- $\left.d_{6^{\prime}} ; 25 \mathrm{MHz}\right), \delta_{\mathrm{C}}: 176.4\left(\mathrm{C}-1^{\prime \prime}\right), 171.3\left(\mathrm{C}-9^{\prime}\right), 153.5\left(\mathrm{C}-3^{\prime}\right), 152.2(\mathrm{C}-5), 141.6$ $\left(\mathrm{C}-1^{\prime}\right), 140.6$ (C-7a), 133.9 (C-4'), 125.7 (C-3a), 125.7 (C-6'), 124.9 (C-2), 123.4 (C-6), 118.9 (C-7), $114.7(\mathrm{C}-4), 112.2(\mathrm{C}-3), 111.1\left(\mathrm{C}-2^{\prime}\right), 105.3\left(\mathrm{C}-5^{\prime}\right), 84.9\left(\mathrm{C}-7^{\prime}\right), 56.1\left(\mathrm{CH}_{3} \mathrm{O}-\right), 55.2\left(\mathrm{C}-8^{\prime}\right), 41.3$ (C-9a; 9b), 39.4 (C-2"), 30.5 (C-8a; 8b), 27.3 (C-3"); HR-ESI-MS m/z: 433.4845 [M - H] (calcd for $\mathrm{C}_{25} \mathrm{H}_{26} \mathrm{~N}_{2} \mathrm{O}_{5}$ 433.4833).

\subsubsection{Serotobenine cyclohexyl ether (2c)}

White solid, (2.5 mg, 20\%). (Scheme 1). Rf $=0.40\left(\mathrm{CHCl}_{3} / \mathrm{MeOH}: 20: 1\right) ;{ }^{1} \mathrm{H}$ NMR (acetone- $d_{\sigma^{\prime}}$; $500 \mathrm{MHz}) ; \delta_{\mathrm{H}}: 10.28(1 \mathrm{H}, \mathrm{br}, \mathrm{H}-1), 8.02(1 \mathrm{H}, \mathrm{br}, \mathrm{H}-10), 7.26\left(1 \mathrm{H}, \mathrm{dd}, J=9.3 \mathrm{~Hz}, \mathrm{H}-6^{\prime}\right), 7.25(1 \mathrm{H}$, d, H-2'), $7.19(1 \mathrm{H}, \mathrm{s}, \mathrm{H}-2), 7.13(1 \mathrm{H}, \mathrm{d}, J=7.0 \mathrm{~Hz}, \mathrm{H}-7), 7.00(1 \mathrm{H}, \mathrm{d}, J=7.0 \mathrm{~Hz}, \mathrm{H}-6) ; 6.69(1 \mathrm{H}, \mathrm{d}$, $\left.J=9.3 \mathrm{~Hz}, \mathrm{H}-5^{\prime}\right), 6.34\left(1 \mathrm{H}, \mathrm{d}, J=10.5 \mathrm{~Hz}, \mathrm{H}-7^{\prime}\right), 4.78\left(1 \mathrm{H}, \mathrm{d}, J=10.5 \mathrm{~Hz}, \mathrm{H}-8^{\prime}\right), 4.16-4.11(1 \mathrm{H}, \mathrm{m}$, $\mathrm{H}-9 \mathrm{~b}), 3.80\left(3 \mathrm{H}, \mathrm{s}, \mathrm{CH}_{3} \mathrm{O}-\right), 3.51-3.49(1 \mathrm{H}, \mathrm{m}, \mathrm{H}-9 \mathrm{a}), 3.50\left(1 \mathrm{H}, \mathrm{m}, \mathrm{H}-1^{\prime \prime}\right) 3.18-3.14(1 \mathrm{H}, \mathrm{m}, \mathrm{H}-8 \mathrm{a})$, $3.05(2 \mathrm{H}, \mathrm{m}, \mathrm{H}-8 \mathrm{~b}), 1.67-1.61\left(4 \mathrm{H}, \mathrm{m}, \mathrm{H}-2^{\prime \prime}\right), 1.54-1.50\left(4 \mathrm{H}, \mathrm{m}, \mathrm{H}-3^{\prime \prime}\right), 1.45-1.39\left(2 \mathrm{H}, \mathrm{m}, \mathrm{H}-4^{\prime \prime}\right)$; ${ }^{13} \mathrm{C}$ NMR (acetone- $\left.d_{6^{\prime}} ; 25 \mathrm{MHz}\right), \delta_{\mathrm{C}}: 171.5\left(\mathrm{C}-9^{\prime}\right), 153.5\left(\mathrm{C}-3^{\prime}\right), 151.9(\mathrm{C}-5), 147.8(\mathrm{C}-7 \mathrm{a}), 136.2$ $\left(\mathrm{C}-1^{\prime}\right), 134.0\left(\mathrm{C}-4^{\prime}\right), 125.8\left(\mathrm{C}-6^{\prime}\right), 125.6(\mathrm{C}-2), 123.8$ (C-3a), 119.6 (C-6), 117.6 (C-7), 115.0 (C-4), $112.2(\mathrm{C}-3), 111.7\left(\mathrm{C}-2^{\prime}\right), 105.4\left(\mathrm{C}-5^{\prime}\right), 85.3\left(\mathrm{C}-7^{\prime}\right), 69.2\left(\mathrm{C}-1^{\prime \prime}\right), 56.2\left(\mathrm{CH}_{3} \mathrm{O}-\right), 55.2\left(\mathrm{C}-8^{\prime}\right), 41.4$ (C-9a; 9b), 29.0 (C-8a; 8b), 24.0 (C-4") 23.8 (C-3"); HR-ESI-MS m/z: 455.4987 [M + Na] (calcd for $\mathrm{C}_{26} \mathrm{H}_{28} \mathrm{~N}_{2} \mathrm{O}_{4} \mathrm{Na}$, 455.5001).

\subsubsection{Zenkerinol (6)}

Orange solid; $[\alpha]_{D}^{25}=-79^{\circ}$ (c 0.3, MeOH); M.p. $295-297^{\circ} \mathrm{C} ; \mathrm{IR}_{\text {umax }}{ }_{\mathrm{KBr}} \mathrm{cm}^{-1}: 3267,2923,2853$, 1595, 1512, 1226, 1159; TLC Rf: $0.42\left(\mathrm{CH}_{2} \mathrm{Cl}_{2} / \mathrm{MeOH}:\right.$ 90/10); ESI-MS m/z: $583.4941[\mathrm{M}+\mathrm{Na}]^{+}$ (calcd for $\mathrm{C}_{30} \mathrm{H}_{24} \mathrm{O}_{11}$ 583.4937); ${ }^{1} \mathrm{H}-\mathrm{NMR}\left(500 \mathrm{MHz}\right.$, DMSO- $\left.d_{6}\right), \delta_{\mathrm{H}}: 6.89\left(2 \mathrm{H}, \mathrm{d}, J=1.7 \mathrm{~Hz}, \mathrm{H}-2^{\prime}\right.$ and $\left.\mathrm{H}-2^{\prime \prime \prime}\right), 6.66\left(2 \mathrm{H}, \mathrm{d}, J=7.2 ; 1.7 \mathrm{~Hz}, \mathrm{H}-6^{\prime}\right.$ and $\left.6^{\prime \prime \prime}\right), 6.65\left(2 \mathrm{H}, \mathrm{dd}, J=7.2 \mathrm{~Hz}, \mathrm{H}-5^{\prime}\right.$ and $\left.\mathrm{H}-5^{\prime \prime \prime}\right)$, $6.18\left(1 \mathrm{H}, \mathrm{s}, \mathrm{H}-6^{\prime \prime}\right), 5.88(1 \mathrm{H}, \mathrm{d}, J=2.3 \mathrm{~Hz}, \mathrm{H}-6), 5.71(1 \mathrm{H}, \mathrm{d}, J=2.3 \mathrm{~Hz}, \mathrm{H}-8), 4.65(1 \mathrm{H}, \mathrm{d}, J=9.5 \mathrm{~Hz}$, $\left.\mathrm{H}-2^{\prime \prime}\right), 3.91\left(1 \mathrm{H}, \mathrm{m}, J=9.5 ; 4.2 \mathrm{~Hz}, \mathrm{H}-3^{\prime \prime}\right), 3.18(1 \mathrm{H}, \mathrm{s}, \mathrm{H}-4), 2.66\left(1 \mathrm{H}, \mathrm{dd}, J=16.7 ; 5.9 \mathrm{~Hz}, \mathrm{H}-4 \beta^{\prime \prime}\right)$, $2.46\left(1 \mathrm{H}, \mathrm{dd}, J=16.7 ; 4.2 \mathrm{~Hz}, \mathrm{H}-4 \mathrm{a}^{\prime \prime}\right) ;{ }^{13} \mathrm{C}-\mathrm{NMR}\left(125 \mathrm{MHz}\right.$, DMSO- $\left.d_{6}\right): \delta_{\mathrm{C}}: 156.8\left(\mathrm{C}-5^{\prime \prime}\right), 156.5^{*}$ 
(C-5), $156.0\left(C-7^{\prime \prime}\right), 156.5^{*}(C-7), 156.0^{*}(C-8 a), 156.5^{*}\left(C-8 a^{\prime \prime}\right), 144.8$ (C-3' and C-3"') 144.7 (C-4' and C-4"'), $140.0(\mathrm{C}-2), 133.1(\mathrm{C}-3), 130.9\left(\mathrm{C}-1^{\prime \prime \prime}\right), 120.2\left(\mathrm{C}-1^{\prime}\right), 118.2\left(\mathrm{C}-6^{\prime}\right.$ and C-6"'), $115.2\left(\mathrm{C}-2^{\prime}\right.$ and C-2"'), $115.2\left(C^{\prime}-5^{\prime}\right.$ and $\left.C-5^{\prime \prime \prime}\right), 102.4\left(C-8^{\prime \prime}\right), 99.9(C-4 a), 99.7\left(C-4 a^{\prime \prime}\right), 98.8\left(C-6^{\prime \prime}\right), 95.4(C-6)$, $94.4(\mathrm{C}-8), 78.3\left(\mathrm{C}-2^{\prime \prime}\right), 65.1\left(\mathrm{C}-3^{\prime \prime}\right), 48.8(\mathrm{C}-4), 28.5\left(\mathrm{C}-4^{\prime \prime}\right) .{ }^{*}$ Signals can be interchanged.

\subsection{Antiplasmodial activity}

Serotobenine derivatives $\mathbf{2} \mathbf{a}$ and $\mathbf{2} \mathbf{b}$ were tested for their antimalarial activity against $P$. falciparum strain 3D7 in a parasite lactate dehydrogenase $(p \mathrm{LDH})$-Assay. The parasites were cultured for $72 \mathrm{~h}$ in $\mathrm{A}+$ human erythrocytes at a haematocrit of $4 \%$ in RPMI media (PAA) supplemented with $10 \% \mathrm{~A}$ + human serum, $200 \mu \mathrm{M}$ hypoxanthine and $20 \mu \mathrm{g} \mathrm{ml}^{-1}$ gentamycin as described previously (Trager \& Jensen 1976) in the presence and absence of the compounds, respectively. Cultures for growth assays were set up at the ring stage of parasites ( $0.1 \%$ parasitemia) in 96 -well plates at a hematokrit of $3 \%$. Parasites were cultured in presence or absence of drugs for up to $72 \mathrm{~h}$. Subsequently cells were harvested, washed and frozen at $-20^{\circ} \mathrm{C}$ until analysis. $p \mathrm{LDH}$ was detected and measured as described elsewhere (Makler et al. 1993; Arnot et al. 2008). Growth was calculated as follow:

$$
G=\frac{O D_{650 \mathrm{~nm}} \mathrm{a}-\mathrm{OD}_{650 \mathrm{~nm}} \mathrm{~b}}{O D_{650 \mathrm{~nm}} \mathrm{c}-\mathrm{OD}_{650 \mathrm{~nm}} \mathrm{~b}} \cdot 100
$$

$a=i R B C$ treated with drugs; $b=i R B C$ treated with cycloheximide; $c=$ non treated $i R B C$ $\mathrm{G}=$ plasmodial growth.

\section{Conclusion}

The genus Campylospermum is a good source of various classes of compounds such as: flavonoids, biflavonoids, ellagic acids, terpenoids, steroids and alkaloids. A large number of antimalarial compounds with a wide variety of structures have been isolated from plants. Among them, several belong to classes mentioned above. Indole alkaloid derivative such as decursivine showed a promising in vitro activity. Structure modification of racemic serotobenine afforded three derivatives nevertheless any of them among the tested compounds do not displayed good activity as the template decursivine.

\section{Acknowledgements}

We gratefully acknowledge the DAAD/STIBET Doktoranden Scholarship for financial support and travel grants for Mr. Mbabi to Germany. The authors are also grateful to the Philipps-Universität Marburg, the Chemistry Department and the Koert's Group for technical assistance. We also thank Mr. Fulbert Tadjouteu (National Herbarium of Cameroon) for his assistance in the collection and identification of the plant material.

\section{Disclosure statement}

No potential conflict of interest was reported by the authors. 


\section{References}

Abouem ZA, Ngono Bikobo DS, Atchadé AT, Mbing NJ, Gangoue PJ, Ghogomu TR, Blond A, Pegnyemb DE, Bodo B. 2008. Nitrile glucosides and serotobenine from Campylospermum glaucum and Ouratea turnarea. Phytochemistry. 69:2209-2213.

Abouem ZA, Ndongo JT, Ngono Bikobo DS, Nkot JL. 2014. Biological studies on nitrogen-containing compounds from Campylospermum Oliveranum and Campylospermum sulcatum (Ochnaceae). Int J Pharm Pharm Sci. 6:252-256.

Arnot DE, Cavanagh DR, Remarque EJ, Creasey AM, Sowa MP, Morgan WD, Holder AA, Longacre S, Thomas AW. 2008. Comparative testing of six antigen-based malaria vaccine candidates directed toward merozoite-stage Plasmodium falciparum. Clin Vaccine Immunol. 15:1345-1355.

Basco L, Mitaku S, Skaltsounis AL, Ravelomanantsoa N, Tillequin F, Koch M, Le Bras J. 1994. In vitro activities of furoquinoline and acridone alkaloids against Plasmodium falciparum. Antimicrob Agents Chemother. 38:1169-1171.

Bayiha Ba Njock G, Bartholomeusz TA, Foroozandeh M, Pegnyemb DE, Christens P, Jeannerat D. 2011. NASCA HMBC, a new NMR methodology for the resolution of severely overlapping signals: application to the study of agathisflavone. Phytochem Anal. 23:126-130.

Bayiha Ba Njock G, Bartholomeusz T, Ngono Bikobo D, Foroozandeh M, Shivapurkar R, Christen P, Pegnyemb DE, Jeannerat D. 2013. Structure and Dynamic of Three Indole Alkaloids from the Campylospermum Genus (Ochnaceae). Helv Chim Acta. 96:1298-1304.

Bouquet A. 1969. Féticheurs et médecine traditionnelles du Congo (Brazzaville) [Fetish and Traditional Medicine of Congo (Brazaville)]. ORSTOM, Paris. 36:177-178.

Cádiz-Gurrea ML, Fernández-Arroyo S, Segura-Carretero A. 2014. Pine bark and green tea concentrated extracts: antioxidant activity and comprehensive characterization of bioactive compounds by HPLCESI-QTOF-MS. Int J Mol Sci. 15:20382-20402.

Chinsembu Kazhila C. 2015. Plants as antimalarial agents in Sub-Saharan Africa. Acta Trop. 152:32-48.

Diarra N, van't Klooster C, Togola A, Diallo D, Willcox M, Jong J. 2015. Ethnobotanical study of plants used against malaria in Sélingué subdistrict Mali. J Ethnopharmacol. 166:352-360.

Frederich M, Tits M, Angenot L. 2008. Potential antimalarial activity of indole alkaloids. T Roy Soc Trop Med Hyg. 102:11-19.

Fukami H, Yano Y, Iwashita T. 2013. Isolation of reduced form of cyanidin-3-O- $\beta$-D-glucopyranoside from immature black soybean (Glycine max (L.) Merr) and its reducing properties. J Oleo Sci. 62:623-629.

Ghogomu R, Sondengam BL, Martin MT, Bodo B. 1987. Lophirone A, A biflavonoid with unusual skeleton from Lophira lanceolata. Tetrahedron Lett. 28:2967-2968.

Ghogomu T, Sondengam BL, Martin MT, Bodo B. 1990. Structure of the Chalcone dimers lophirone F, $\mathrm{G}$ and $\mathrm{H}$ from Lophira lanceolata stem bark. Phytochemistry. 29:2289-2293.

Klika KD, Ricarte I, Salles Trevisan MT, Vasconcelos Silva MGD, Owen RW. 2015. (2R, 3S, 4S, 2"R,3"S)Guibourtinidol-( $4 \alpha \rightarrow 8)$-catechin, a biflavonoid procyanidin of the proguibourtinidin group from Senna macranthera: its relative stereochemistry and conformation. Tetrahedron. 26:247-250.

Makler MT, Ries JM, Williams JA, Bancroft JE, Piper RC, Gibbins BL, Hinrichs DJ. 1993. Parasite lactate dehydrogenase as an assay for Plasmodium falciparum drug sensitivity. Am J Trop Med Hyg. 48(6):739-741.

Malan EM, Swinny E, Ferreirat D, Steynberg P. 1996. The structure and synthesis of proguibourtinidins from Cassia abbreviata. Phytochemistry. 41:1209-1213.

Marković JMD, Marković Z, Krstić JB, Amic D. 2013. Interpretation of the IR and Raman spectra of morin by density functional theory and comparative analysis. Vibr Spect. 64:1-9.

Mashima T, Okigawa M, Kawano N, Khan NU, llyas M, Rahman W. 1970. On the bisflavones in the leaves of agathis alba foxworthy. Tetrahedron Lett. 33:2937-2940.

Mello de J C P, Petereit F, Nahrstedt A. 1999. A dimeric proanthocyanidin from Stryphnodendron adstringens. Phytochemistry. 51:1105-1107.

Messanga BB, Ghogomu R, Sondengam BL, Martin MT, Blond A, Brouard JP, Bodo B. 1998. Calodenin C: a new guibourtinidoi-( $4 \alpha \rightarrow 8)$-afzelechin from Ochna calodendron. Planta Med. 64:760-761. 
Ndongo JT, Shaaban M, Ngo Mbing J, Ngono Bikobo DS, Atchadé AT, Pegnyemb DE, Laatsch H. 2010. Phenolic dimers and an indole alkaloid from Campylospermum flavum (Ochnaceae). Phytochemistry. 71:1872-1878.

Ndongo JT, Issa ME, Messi NA, Ngo Mbing J, Cuendet M, Pegnyemb DE. 2015. Cytotoxic flavonoids and other constituents from the stem bark of Ochna schweinfurthiana (Ochnaceae). Nat Prod Res. 29(17):1684-1687.

Ngo Mbing J, Balemaken MM, Ndongo JT, Bayiha Ba Njock G, Atchadé AT, Pegnyemb DE. 2014. New flavonoids C-glycosides from Rhabdophyllum arnoldianum (Ochnaceae). Nat Prod Res. 28:539-544.

Ngono Bikobo DS, Nkot JL, Mosset P, Atchadé AT, Ndongo JT, Pemha R, Pegnyemb DE. 2011. Acylsteryl glycosides and other constituents from Campylospermum densiflorum (Ochnaceae). Rasayan J Chem. 4:753-763.

Ngono Bikobo DS, Mosset $\mathrm{P}$, Abouem A, Zintchem A, Atchadé AT, Balemaken Missi M, Mbabi Nyemeck II, N, Pegnyemb DE. 2014. Campylospermine, an N-Hydroxy-alkaloid from the leaves of Campylospermum densiflorum (Ochnaceae). Int J Phyto Pharm Res. 6:719-728.

Ngono Bikobo DS, Zintchem Abouem A, Zintchem A, Mbabi Nyemeck II, N, Atchadé AT, Bayiha Ba Njock G, Mosset P, and Pegnyemb DE. 2015. Secondary metabolites from Campylospermum oliverianum (Farron), Campylospermum glaucum (Tiegh) and Campylospermum dybowskii (Van Tiegh). Int J Phyto Pharm Res. 7(1):119-127.

Park HM, Mi-Won S, Donghyun K, Seon-Hee K, Sung-Hoon K, Hak CK, Sun YK. 2011. Fatty acid components of hardy kiwi fruit (actinidia arguta) as II-4 production inhibitor. Biomol Ther. 19:126-133.

Qin H, Xu Z, Cui Y, Jia Y. 2011. Total synthesis of decursivine and serotobenine: a Witkop photocyclization/ elimination/O-Michael Addition cascade approach. Angew Chem Int Ed. 50:4447-4449.

Ragab EA, Mohammed AESI, Abbass HS, Kotb SI. 2013. A new flavan-3-ol dimer from Ficus spragueana leaves and its cytotoxic activity. Pharmacogn Mag. 9:144-148.

Sato H, Kawagishi H, Nishimura T, Yoneyama S, Yoshimoto Y, Sakamura S, Furusaki A, Katsu S. 1985. Serotobenine, a Novel Phenolic Amide from Safflower Seeds (Carthamus tinctorius L.). Agr Biol Chem Tokyo. 49:2969-2974.

Taniguchi S, Kuroda K, Yoshikado N, Doi KI, Tanabe M, Shibata T, Yoshida T, Hatano T. 2007. New dimeric flavans from Gambir, an extract of Uncaria Gambir. Heterocycles. 74:595-605.

Trager W, Jensen JB. 1976. Human malaria parasites in continuous culture. Science. 193:673-675.

Wang CM, Hsu YM, Jhan YL, Tsai SJ, Lin SX, Su SH, Chou CH. 2015. Structure elucidation of procyanidins isolated from rhododendron formosanum and their anti-oxidative and anti-bacterial activities. Molecules. 20:12787-12803.

White NJ. 2004. Anti-malarial drug resistance. J Clin Invest. 113:1084-1092.

World Health Organization. 2014. World malaria report 2013. Geneva, Switzerland: World Health Organization; [cited 2015 Apr 14]. Available from: http://www.WHO.int/malaria/publications/ world_malaria_report_2013/en/.

Zhang H, Qui S, Tamez P, Tan GT, Aydogmus Z, Van Hung N, Cuong NM, Angerhofer C, Soejarto DD, Pezzuto JM, Fong HHS. 2002. Antimalaria agents from plants II. Decursivine, a new antimalarial indole alkaloid from Rhaphidophora decursiva. Pharm Biol. 40:221-224. 